\title{
El reto de sostener cultura de la seguridad del paciente en las instituciones sanitarias
}

\section{The challange of upholding the culture of patient safety in the health institutions}

doi.org/10.23938/ASSN.0018

M.P. Astier-Peña ${ }^{1}$, G. Olivera-Cañadas ${ }^{2}$

La Organización Mundial de la Salud (OMS), en el lanzamiento de su Alianza Mundial para la Seguridad del Paciente (AMSP) ${ }^{1}$ definió la Seguridad del Paciente (SP) como la "ausencia de daño prevenible al paciente durante el proceso de la atención a la salud". El informe Crossing the quality chasm: a new health system for the 21st Century ${ }^{2}$ pone de manifiesto que la calidad asistencial de los pacientes se ve alterada cuando se producen eventos adversos (EA) que además son prevenibles. Es por esto que la SP ha adquirido un papel protagonista en la gestión de la calidad de los servicios sanitarios, y la OMS y otras organizaciones ${ }^{3}$ vinculan explícitamente la SP con la calidad de la atención sanitaria y recomiendan que se preste la mayor atención posible a este aspecto, como refleja el informe To err is human, building a safer health system $^{4}$, que mide por primera vez la trascendencia de una forma global y el impacto de los problemas de SP en el sistema sanitario.

La calidad y la SP son culturas estrechamente vinculadas, de forma que no se podría hablar enteramente de la primera sin considerar el enfoque de la segunda. Se trata, en definitiva, de mejorar la calidad de la atención sanitaria mediante la incorporación de intervenciones y estrategias de SP. Pero la calidad asistencial en las instituciones sanitarias está estrechamente relacionada al grado de compromiso de los profesionales que trabajan en estas instituciones; sin el compromiso de los profesionales no hay calidad ni seguridad. Es, por tanto, crucial que en las organizaciones sanitarias se cultive la calidad y la seguridad y que los profesionales que en ellas trabajan refieran una percepción óptima de la calidad y la seguridad en sus entornos de trabajo.

Y ¿cómo definimos y medimos esa cultura de calidad y seguridad? Se define cultura de seguridad de una organización como la suma de

1. Médico de familia. Centro de Salud de Tauste (Zaragoza). Coordinadora del grupo de seguridad del paciente de la Sociedad Española de Medicina Familiar y Comunitaria.

2. Médico de familia. Directora de Continuidad Asistencial. Hospital Universitario San Carlos. Madrid. Miembro del grupo de seguridad del paciente de la Sociedad Española de Medicina Familiar y Comunitaria.

\section{Correspondencia:}

$M^{\text {a }}$ Pilar Astier-Peña

Centro de Salud de Tauste (Zaragoza)

mpastier@gmail.com 
valores, actitudes, percepciones, competencias y patrones de conducta individuales y grupales que determinan el estilo, la competencia y el compromiso de la gestión de la seguridad en una organización. Las organizaciones con una cultura de seguridad positiva se caracterizan por una comunicación basada en la confianza mutua, comparten la percepción de la importancia de la seguridad y confían en la eficacia de las acciones preventivas ${ }^{5}$. Tanto las personas como la organización son capaces de reconocer errores, aprender de ellos, y actuar para su rectificación. La cultura de SP, según la definición adoptada en 2006 por la European Society for Quality in Health Care, busca continuamente minimizar el daño al paciente que puede resultar del proceso de prestación de la asistencia sanitaria, introduciendo el concepto de cultura dinámica con las acciones adoptadas para reducir el daño ${ }^{6}$.

La cultura de seguridad se considera fundamental para las prestaciones de seguridad en numerosas industrias de alto riesgo, como la nuclear, la química, la petrolera, la ferroviaria y la de la aviación. Las organizaciones que prestan asistencia sanitaria, dada la elevada morbilidad y mortalidad que conlleva dicha prestación, son consideradas como industrias de alto riesgo, es por ello que la mejora de la cultura de seguridad se convierte en una acción prioritaria?.

Algunos de los beneficios esperados del desarrollo de una cultura de seguridad en las instituciones sanitarias son:

La reducción potencial en la recurrencia y la gravedad de incidentes de SP, a través del aumento de notificación y aprendizaje organizativo, gracias a los sistemas de notificación.

La reducción del daño físico y psicológico que los pacientes puedan sufrir, gracias al trabajo realizado para prevenir errores, sobre todo facilitando el hablar de forma abierta cuando las cosas fallan.

La mejora en la gestión de recursos gracias a una evaluación eficaz del riesgo y al cambio de las prácticas en la atención sanitaria como consecuencia de los incidentes analizados.

La reducción en los costes financieros y sociales provocados por incidentes de SP, incluyendo pérdida de tiempo laboral y prestaciones por invalidez.

En 2003, el National Quality Forum ${ }^{8}$ identificó como la primera de sus "30 prácticas seguras" promover una cultura de seguridad en la organización, entendiendo que el mayor cambio, para pasar a un sistema sanitario más seguro, es cambiar a otra cultura en la que los incidentes no sean tratados como fallos de las personas sino como oportunidades para mejorar el sistema y prevenir el daño. Esta fue también una de las conclusiones de la Declaración del Consejo de Europa en Varsovia, en esta declaración se insta a los países miembros a trabajar en mejorar la cultura de SP en sus organizaciones para abordar el problema a escala nacional.

En nuestro pais, el Plan de Calidad para el Sistema Nacional de Salud 2006-2010 contemplaba entre las grandes áreas de interés sanitario el fomento de la excelencia clínica y formulaba como una de las estrategias para lograrla, la estrategia $n^{\circ}$ 8: mejorar la seguridad de los pacientes atendidos en los centros sanitarios del SNS. El primer objetivo de esta estrategia ${ }^{9}$ no era otro que promover una cultura de la seguridad en todos los niveles de la atención sanitaria desde un enfoque de la gestión proactiva, preventiva y del aprendizaje. 
Evaluar la cultura de seguridad en una organización implica saber lo que se "dice" al respecto de la misma y valorar lo que se "hace". Entre ambas cuestiones generalmente suele existir disparidad. A esta disparidad hay que añadir la creencia real que el personal tiene sobre cómo su organización valora la seguridad y la percepción que afecta a su propio comportamiento. La evaluación de las creencias y percepciones son importantes ya que afectan al propio comportamiento $\mathrm{y}$, por tanto, tienen consecuencias en la seguridad. Para hacer esta evaluación de lo que es la cultura de seguridad, existe una amplia variedad de instrumentos tanto cualitativos como cuantitativos, siendo los cuestionarios la herramienta utilizada con mayor frecuencia ${ }^{10}$, ya que posibilitan el obtener una imagen general de las creencias, valores y normas relacionadas con la seguridad que comparten los miembros de la organización. Con ellos se consigue evaluar el nivel de cultura grupal en SP como un indicador intermedio fiable y específico de los niveles de seguridad de un servicio asistencial concreto ${ }^{11}$. Mediante los cuestionarios se pueden identificar puntos fuertes y áreas de mejora de la cultura de seguridad y se puede desarrollar un plan de acción que contribuya a la mejora de la seguridad.

Las organizaciones sanitarias han considerado de gran importancia la SP y, en relación a este concepto, los factores culturales a promover dentro de la organización como favorecedores de un buen clima de seguridad. Esto ha propiciado el desarrollo de investigaciones sobre instrumentos que puedan medir el cambio cultural que facilite la implantación y consecución de mejoras en la seguridad de los pacientes ${ }^{12}$.

La mayoria de estos cuestionarios para medir la cultura de seguridad son adaptaciones de los utilizados en los sectores industriales. A este respecto, existen varias revisiones que sintetizan los más utilizados para medir este cambio cultural y por tanto la mejora en la SP: Colla y col $(2005)^{12}$, Flin y col $(2006)^{7}$ y la revisión de Halligan $(2011)^{13}$ que es una de las más recientes y que además aporta un conjunto común tanto de definiciones como de dimensiones que pueden ayudar a los investigadores a compartir mejor la información y las estrategias para mejorar la cultura de seguridad. Además en 2010, la iniciativa EUNetPaS, European Network for Patient Safety, recopiló e identificó treinta cuestionarios diferentes para medir cultura de SP en el ámbito sanitario ${ }^{13}$, siendo de todos ellos los más utilizados: el Hospital Survey on Patient Safety (HSOPS) ${ }^{14}$, el Medical Office Survey on Patient Safety (MOSPS) ${ }^{15}$, el Safety Attitudes Questionnaire (SAQ) ${ }^{16}$, el Safety Climate Survey (SCS) ${ }^{17}$, el Manchester Patient Safety Assessment Framework (MaPSaF) ${ }^{18}$ y el $\mathrm{Pa}$ tient Safety Culture in Healthcare Organizations (PSCHO) ${ }^{19}$. Con respecto a las caracteristicas más destacables habría que mencionar que todos son fundamentalmente de ámbito general, su cumplimentación es individual excepto el MaPSaF que se contesta en grupo; el número de ítems varía desde 10 hasta casi 80 y las respuestas se establecen según una escala de medida tipo Likert, que permite valorar el grado de acuerdo con el enunciado del ítem; el número de dimensiones que contienen oscila entre 4 y 16 y todos incluyen la notificación de incidentes de seguridad relacionados con la SP como un ítem más.

La evaluación periódica de la cultura de seguridad es útil para conocer el estado y la evolución de las percepciones de los profesionales y ayuda en la elaboración de planes de mejora. La periodicidad en la 
realización de estas encuestas debe ser suficiente para que las medidas adoptadas hayan tenido tiempo para su implantación efectiva y los profesionales hayan percibido sus resultados. Sin embargo, el grado de seguridad percibido de manera global puede ser un indicador poco sensible para el seguimiento de la eficacia de las acciones implementadas como así ocurre en el estudio publicado en este número de la revista sobre Evolución de la cultura de seguridad del paciente en un hospital de media-larga estancia: indicadores de seguimiento ${ }^{20}$ en el que se presentan los resultados de medición de la cultura en un hospital, en dos ocasiones, y muestra que el grado de seguridad percibida de forma global es similar y adecuado en ambos cortes, aunque al analizar las distintas dimensiones de forma individualizada, sí que se observan diferencias entre ambos estudios y se detectan las mejoras realizadas. Estas mejoras afectan directamente a dimensiones individuales por cambios, entre otros, del equipo directivo e intervenciones organizacionales que pudieron modificar las percepciones del personal. En cualquier caso, podemos concluir que la medición periódica y el análisis de la cultura de seguridad del paciente permite conocer cómo es la percepción de los profesionales en relación con la SP y es una manera de mantener en el tiempo de forma sostenible dicha cultura de SP en las instituciones sanitarias.

\section{BIBLIOGRAFÍA}

1. World Health Organization [Internet]. Geneva: World Health Organization (WHO); 2016 [consultado 19 Nov 2016]. Patient safety. World Alliance for Patient Safety. The Launch of the World Alliance for Patient Safety, Washington DC, USA. 27 de octubre 2004. Disponible en: http://www.who.int/patientsafety/worldalliance/en/

2. Institute of Medicine (US) Committee on Quality of Health Care in America. Crossing the Quality Chasm: A New Health System for the 21st.

3. Organización Mundial de la Salud (OMS). 55 asamblea Mundial de la Salud WHA A55/52. Punto 13.9 del orden del día 18 de mayo de 2002. Calidad de la atención: seguridad del paciente [Internet]. Ginebra: Organización Mundial de la Salud [consultado Oct 2016]. Disponible en: http://apps.who.int/gb/ archive/pdf_files/WHA55/sa5552.pdf

4. L. T. Kohn, J. M. Corrigan, and M. S. Donaldson, Eds. Washington, D.C: The National Academies Press; 2000. doi: 10.17226/9728 [consultado en octubre de 2016]. Disponible en: http://www.nap.edu/catalog/9728.html

5. NIEVA VF, SORRA J. Institute of Medicine. To err is human: building a safer health system. Safety culture assessment: a tool for improving patient safety in healthcare organizations. Qual Saf Health Care 2003; 12 (suppl II): ii17-ii23.

6. European Union Network for Patient Safety. Use of Patient Safety Culture Instruments and Recommendations [Internet]. Aarhus: European Society for Quality in Healthcare, Office for Quality Indicators; 2010 [consultado el 30 de octubre de 2016]. Disponible en: http://www.seguridaddelpaciente.es/ resources/documentos/2016/eunetpas/WP1-REPORT\%20\%20Use $\% 20$ of $\% 20$ \%20PSCI\%20and\%20recommandations\%20-\%20March\%20\%202010.pdf

7. Flin R, Burns C, Mearns K, Yule S, Robertson EM. Measuring safety climate in health care. Qual Saf Health Care 2006; 15: 109-115.

8. The National Quality Forum. Safe Practices for Better Healthcare. A Consensus Report [monografía en Internet]. Washington, DC: The National Quality Forum; 2003 [consultado el 28 de octubre de 2016]. Disponible en: https:// archive.ahrq.gov/professionals/quality-patient-safety/patient-safety-resources/resources/nqfpract/nqfpract.pdf. 
9. Agra Y, Terol E. La seguridad del paciente: una estrategia del Sistema Nacional de Salud. An Sis Sanit Navar 2006; 29: 319-323.

10. O'Connor P, Buttrey SE, O'Dea A, Kennedy Q. Identifying and addressing the limitations of safety climate surveys. J Safety Res 2011; 42: 259-265.

11. Ruiz Sánchez M, Borrell-Carrió F, Ortodó Parra C, Fernàndez I Danés N, Fité GaLLEGo A. Auditorías en seguridad clínica para centros de atención primaria. Estudio piloto. Aten Primaria 2013; 45: 341-348.

12. Colla JB, Bracken AC, KinNey LM. Measuring patient safety climate: a review of surveys. Qual Saf Health Care 2005; 14: 364-366.

13. European Union Network for Patient Safety. Patient Safety Culture Instruments used in Member States. Aarhus: European Society for Quality in Healthcare, Office for Quality Indicators; 2010.

14. Sorra J, Franklin M, Streagle S. Survey User's Guide. Hospital Survey on Patient Safety Culture. Rockville, MD: Agency for Healthcare Research and Quality (AHRQ); 2008.

15. Medical Office Survey on Patient Safety Culture. Content last reviewed November 2016. Agency for Healthcare Research and Quality, Rockville, MD. [consultado el 31 de octubre de 2016]. Disponible en: http://www.ahrq.gov/ professionals/quality-patient-safety/patientsafetyculture/medical-office/index.html

16. Sexton JB, Helmreich RL, Neilands TB, Rowan K, Vella K, Boyden J et al. The safety attitudes questionnaire: psychometric properties, benchmarking data, and emerging research. BMC Health Serv Res 2006; 6: 44.

17. Sexton J, Thоmas E. The safety climate survey: psychometric and benchmarking properties. Austin, TX: The University of Texas Center of Excellence for Patient Safety Research and Practice; 2003.

18. National Patient Safety Agency, National Health Service. Manchester Patient Safety Framework (MaPSaF). Primary Care: The University of Manchester; 2006 [consultado el 21 de noviembre de 2016]. Disponible en: http://www. nrls.npsa.nhs.uk/resources/?entryid45=59796.

19. Singer S, Meterko M, Baker L, Gaba D, Falwell A, Rosen A. Patient Safety Climate in Healthcare Organizations (PSCHO). Measurement Instrument Database for the Social Science [Internet]. Galway; 2012 [consultado el 21 de noviembre de 2016]. Disponible en: http://www.midss.org/sites/default/files/pscho_ survey_2006.pdf

20. Merino-Plaza MJ, Carrera-Hueso FJ, Castillo-Blasco M, Martínez-Asensi A, MartíNEZ-CAPELLA R, FRIKI-BENBRAHIM N. Evolución de la cultura de seguridad del paciente en un hospital de media-larga estancia: indicadores de seguimiento. An Sist Sanit Navar 2017; 41: 43-56. 
